\title{
ACTITUDES Y ESTILOS DE VIDA DE LOS CONSUMIDORES DE ALIMENTOS ECOLÓGICOS EN EL MERCADO DE MADRID (ESPAÑA)
}

\author{
$\underline{\text { Rodolfo Bernabéu }}^{{ }^{a}}$, Mónica Díaz ${ }^{\text {b }}$ Adrián Rabadán ${ }^{a}$ \\ ${ }^{a}$ Escuela Técnica Superior de Ingenieros Agrónomos y de Montes (ETSIAM) de Albacete \\ (Rodolfo.Bernabeu@uclm.es)(Adrian.Rabadan@uclm.es). Universidad de Castilla-La Mancha. \\ ${ }^{b}$ Escuela Técnica Superior de Ingenieros Agrónomos (ETSIA) de Ciudad Real (Monica.Diaz@uclm.es). \\ Universidad de Castilla-La Mancha
}

\section{Resumen}

La producción de alimentos ecológicos en España se incrementa año tras año como respuesta a las demandas sociales en materia de alimentación y salud, sobre todo en lo que se refiere a los alimentos seguros, nutritivos y sostenibles. No obstante, ese incremento en la producción no va acompañado por un incremento similar en su consumo en España, por lo que gran parte de la producción se exportan a otros países, fundamentalmente de la Unión Europea. Para intentar incrementar su consumo, el presente trabajo identifica, en función del grado de conocimiento que tienen los consumidores sobre los alimentos ecológicos, sus motivos de compra y estilos de vida con el fin de dirigir distintas acciones comerciales por los agentes del sector. Para ello, se realizaron 415 encuestas en diciembre de 2017 y enero de 2018 en los alrededores de las principales zonas comerciales de Madrid capital. El muestreo se realizó de forma aleatoria y estratificada, por género y grupo de edad, para un nivel de error inferior al $5 \%$ y un nivel de confianza al $95,5 \%$.

Palabras clave: Consumo, Encuestas, Marketing agroalimentario, Salud, Sostenibilidad.

\section{Introducción y objetivos}

La Comisión Europea presentó en 2018 sus propuestas legislativas sobre la Política Agraria Común (PAC) durante el periodo 2021-2027 que tienen como fin el seguir prestando un firme apoyo a la agricultura europea, favorecer la prosperidad de las zonas rurales y producir alimentos de calidad, así como realizar una contribución significativa al Pacto Verde Europeo (Comisión Europea, 2019).

Dentro de las propuestas, se encuentra como medida transversal la potenciación de la agricultura ecológica, que tiene como fin el promover cambios en las prácticas agrícolas que entrañen una aportación positiva a la producción de alimentos de calidad y a la seguridad alimentaria, al medio ambiente y al clima, al desarrollo sostenible y a la eficiencia en el uso de los recursos naturales, su reutilización y la disminución de los residuos generados, dentro del marco de la economía circular y de la bioeconomía.

Dentro de la UE, España es el país que más superficie dedica a la agricultura ecológica, alcanzando en el año 2018 unas 2.246.475 ha, Francia se sitúa en segundo lugar con 2.035.024 ha, Italia en tercer lugar con 1.958.045 ha, Alemania en cuarto lugar con 1.521.314 ha y Austria, en quinto lugar, con 637.805 ha.

En 2018, las ventas al por menor de alimentos ecológicos en el Mundo fueron de 97.000 millones de $€$ y un gasto medio de 12,9 €/per capita (si bien con gran variabilidad pues en Suiza y Dinamarca, el gasto medio supone $312 € /$ per capita; Suecia, $231 € /$ per capita; Luxemburgo, $221 € /$ per capita; Austria, $205 € /$ per capita; Noruega, $159 € /$ per capita; Francia, $136 € /$ per capita; Alemania $132 € /$ per capita; USA, $125 € /$ per capita y Arabia Saudí, $93 €$ /per capita, entre otros) (FiBL e INFOAM, 2020).

En 2016, las ventas al por menor de alimentos ecológicos en España fueron de 1,686 millones de $€$, con un crecimiento interanual de ventas del $12.6 \%$, con una cuota de mercado del $1,69 \%$ y un gasto medio del consumidor de 36,33 €/per capita (Ecological, 2018).

No obstante, ante la gran disparidad en términos de producción y consumo de alimentos ecológicos en España reflejada en sus cifras de venta, resulta prioritario caracterizar al consumidor de alimentos ecológicos con el fin de orientar al sector productor y en particular, al de Castilla-La Mancha, el presente trabajo persigue determinar cuáles son los principales atributos que los consumidores valoran a la hora de adquirir alimentos ecológicos y cuando no los adquieren, cuáles son los principales motivos así como sus estilos de vida de los consumidores y su grado de conocimiento sobre los alimentos ecológicos.

\section{Metodología}

Para la obtención de los datos se ha encuestado a 415 consumidores de Madrid capital entre el 15 de diciembre de 2017 y el 15 de enero de 2018. En el diseño de la muestra se utilizaron los datos de población 
de Madrid del año 2016 según el Instituto Nacional de Estadística (INE, 2017). El muestreo se realizó de forma aleatoria y estratificada, por género y grupo de edad (18 y 34 años, 35 y 64 años y mayor o igual a 65 años). El error muestral fue inferior al 5\% para un nivel de confianza al 95,5\% ( $\mathrm{p}=\mathrm{q}=0,5 ; \mathrm{k}=2)$ (Cuadro 1).

Cuadro 1. Ficha técnica

\begin{tabular}{ll}
\hline Ámbito & Madrid capital \\
Universo & Consumidores mayores de edad \\
Tamaño muestral & 415 encuestas \\
Error muestral & $<( \pm 5 \%)$ \\
Nivel de confianza & $95,5 \%(\mathrm{k}=2)$ \\
Muestreo & Aleatorio estratificado con afijación proporcional por \\
Control & género y edad \\
Cuestionario previo & De coherencia y estabilidad \\
Trabajo de campo & Pretest a 25 personas \\
\hline
\end{tabular}

Para determinar el grado de conocimiento que los consumidores tienen sobre los alimentos ecológicos se les pidió que respondieran si estaban de acuerdo o no, sobre si son alimentos ecológicos son: a) alimentos de huerta que se venden directamente en mercadillos en plazas, b) alimentos denominados naturales o verdes, c) alimentos cultivados o elaborados sin utilizar productos químicos de síntesis, d) alimentos integrales, e) alimentos sin conservantes ni colorantes ni aditivos artificiales y f) alimentos con sello de certificación de agricultura ecológica. Los entrevistados debian contestar a un máximo de dos ítems. Se consideró que los consumidores tenían un conocimiento alto sobre los alimentos ecológicos aquellos que contestaron afirmativamente y de forma simultánea los ítems c) y f) mientras que el resto de consumidores se consideraron que tenían un grado de conocimiento medio-bajo.

Con estos dos grupos de consumidores, se realizó el test de Levene y un anáisis de la varianza para determinar la existencia o no de diferencias significativas entre de distintas afirmaciones sobre la importancia de los atributos de los alimentos ecológicos y los motivos de no compra de alimentos ecológicos. Adicionalmente, se les preguntó distintas afirmaciones sobre sus estilos de vida, que respondieron en función de su grado de acuerdo/desacuerdo mediante una escala de Likert de cinco puntos (siendo el valor 1 cuando está muy en desacuerdo y el valor 5 cuando está muy de acuerdo).

El procesamiento informático de la información se realizó con el paquete estadístico SPSS para Windows, versión 22.0 (SPSS, 2013).

\section{Resultados}

Los consumidores de alimentos que se consideraron con un conocimiento alto sobre la producción de alimentos ecológicos fueron el $43,6 \%$ de la muestra.

Con relación a la importancia que los consumidores otorgan a los atributos a la hora de adquirir un alimento ecológico, declaran que son beneficiosos para la salud (19,7\%), el respeto al medio ambiente $(18,2 \%)$, la ausencia de conservantes y colorantes $(15,4 \%)$, el sabor $(14,5 \%)$, el valor nutricional $(10,1 \%)$, el precio $(9,6 \%)$, la zona donde ha sido producido o cultivado $(8,1 \%)$, la denominación de marca $(2,3 \%)$ y el aspecto exterior (2,2\%). En función del grado de conocimiento de los consumidores (Cuadro 2), con diferencias significativas, aquellos que tienen un conocimiento alto con respecto a los de un conocimiento medio-bajo, tienen menos en cuenta el precio $(\mathrm{P}<0,1)$ y más el respeto al medio ambiente $(\mathrm{P}<0,01)$.

Los motivos generales de no comprar alimentos ecológicos son por cuestiones relacionadas con el establecimiento (dificultades para locarlizarlos o no encontrarlos dentro del establecimiento o bien no saber en qué establecimiento se venden) (49,3\%), tener un precio elevado $(24,5 \%)$, difícil conservación $(13,85)$, no encontrar diferencias con los alimentos convencionales $(8,2 \%)$ y no gustar su aspecto exterior $(4,2 \%)$. En función del grado de conocimiento de los consumidores (Cuadro 3), en general se observa que con diferencias significativas, aquellos que tienen un conocimiento alto con respecto a los de un conocimiento medio-bajo, tienen menos en cuenta que sean de difícil conservación $(\mathrm{P}<0,1)$.

Con relación a los estilos de vida de los consumidores y en función del grado de conocimiento de los consumidores (Cuadro 4), en general se observa que con diferencias significativas, aquellos que tienen un conocimiento alto con respecto a los de un conocimiento medio-bajo, procuran reducir el estrés y llevar una vida metódica y ordenada, y leer las etiquetas de los alimentos $(\mathrm{P}<0,01)$, controlan la ingesta de sal y prefieren consumir productos reciclados $(\mathrm{P}<0,05)$ y procuran comer alimentos sin aditivos $(\mathrm{P}<0,1)$. 
Cuadro 2. Importancia de los atributos de los alimentos ecológicos en función del grado de conocimiento de los consumidores

\begin{tabular}{lcc}
\hline \multicolumn{1}{c}{ Variable } & $\begin{array}{c}\text { Grado de conocimiento de los alimentos } \\
\text { ecológicos }\end{array}$ \\
& Alto & $\begin{array}{c}\text { Medio-bajo } \\
(56,4 \%)^{1}\end{array}$ \\
\hline Precio* & $(43,6 \%)^{1}$ & 1,59 \\
Sabor & 1,67 & 1,41 \\
Denominación de marca & 1,48 & 1,91 \\
Beneficioso para la salud & 1,91 & 1,26 \\
Aspecto exterior & 1,20 & 1,91 \\
Ausencia de conservantes y colorantes & 1,93 & 1,43 \\
Valor nutricional & 1,36 & 1,59 \\
Respeto al medio ambiente*** & 1,62 & 1,36 \\
Zona de producción & 1,20 & 1,66 \\
\hline
\end{tabular}

${ }^{1}$ Tamaño del segmento.

* y*** Indican diferencias significativas con un máximo nivel de error del $10 \%$ y 1\%, respectivamente.

Cuadro 3. Motivos de no compra de alimentos ecológicos en función del grado de conocimiento de los consumidores

\begin{tabular}{lcc}
\hline \multicolumn{1}{c}{ Variable } & $\begin{array}{c}\text { Grado de conocimiento de los alimentos } \\
\text { ecológicos }\end{array}$ & $\begin{array}{c}\text { Medio-bajo } \\
(56,4 \%)^{1}\end{array}$ \\
& $\begin{array}{c}\text { Alto } \\
(43,6 \%)^{1}\end{array}$ & 1,26 \\
Precio elevado & 1,19 & 1,40 \\
Difícil localizarlos en el establecimiento & 1,43 & 1,43 \\
No se encuentran en el establecimiento donde compro & 1,43 & 1,68 \\
No sé en qué establecimiento se venden & 1,69 & 1,75 \\
No encuentro diferencias con los alimentos convencionales & 1,81 & 1,87 \\
No me gusta su aspecto exterior & 1,90 & 1,58 \\
Son de difícil conservación* & 1,67 & \\
\hline
\end{tabular}

${ }^{I}$ Tamaño del segmento.

* Indica diferencias significativas con un máximo nivel de error del 10\%.

Cuadro 4. Estilos de vida de los consumidores en función del grado de conocimiento de los alimentos ecológicos

\begin{tabular}{lcc}
\hline \multicolumn{1}{c}{ Variable } & $\begin{array}{c}\text { Grado de conocimiento de los alimentos } \\
\text { ecológicos }\end{array}$ \\
& Alto & $\begin{array}{c}\text { Medio-bajo } \\
(56,4 \%)^{1}\end{array}$ \\
\hline Controlo la ingesta de sal** & $(43,6 \%)^{1}$ & 3,50 \\
Practico una dieta vegetariana & 3,76 & 1,88 \\
Hago ejercicio con regularidad & 1,96 & 3,50 \\
Procuro no comer alimentos procesados & 3,48 & 3,26 \\
Como con frecuencia frutas y verduras & 3,41 & 4,02 \\
Como con moderación carne roja & 4,14 & 3,56 \\
Pertenezco a una asociación de defensa de la naturaleza & 3,61 & 1,84 \\
Procuro comer alimentos sin aditivos* & 1,77 & 3,05 \\
Periódicamente chequeo mi salud & 3,29 & 3,28 \\
Procuro reducir el estrés*** & 3,33 & 3,24 \\
Colaboro con ONGs & 3,56 & 2,63 \\
Visito al dentista con regularidad & 2,88 & 3,34 \\
Procuro llevar una vida ordenada y metódica*** & 3,43 & 3,54 \\
Procuro equilibrar trabajo con vida privada & 3,83 & 3,70 \\
Leo las etiquetas de los alimentos*** & 3,86 & 3,62 \\
Prefiero consumir productos reciclados** & 3,93 & 3,50 \\
Arrojo la basura en contenedores selectivos & 3,75 & 4,08 \\
\hline
\end{tabular}

${ }^{1}$ Tamaño del segmento.

* Indica diferencias significativas con un máximo nivel de error del 10\%. 


\section{Conclusiones}

La principal limitación del consumo de alimentos ecológicos es la disponibilidad de los mismos en los establecimientos. La mayor proporción de consumidores con conocimientos sobre los alimentos ecológicos hace que no valoren tanto el precio y si que sean respetuosos con el medio ambiente, puesto de manifiesto a su vez con el estilo de vida del consumidor.

\section{Agradecimientos}

Los autores desean agradecer la financiación concedida al Grupo de Investigación en "Economía Agroalimentaria y Forestal. Bioeconomía" por el Vicerrectorado de Investigación y Política Científica, Programa 541 A, del presupuesto para 2020 de la Universidad de Castilla-La Mancha y cofinanciada por el Fondo Europeo de Desarrollo Regional.

\section{Bibliografía}

Comisión Europea (2019). The European Green Deal. Disponible en: https://eurlex.europa.eu/resource.html?uri=cellar:b828d165-1c22-11ea-8c1f-

01aa75ed71a1.0004.02/DOC_1\&format=PDF (Consulta realizada el 7 de febrero de 2021).

Ecological (2018). El sector ecológico en España 2018. Disponible en: https://www.ecological.bio/es/sectorbio2018/El-Sector-Ecologico-en-Espa\%C3\%B1a-2018-

EcoLogical.pdf (Consulta realizada el 14 de diciembre de 2020).

FiBL e INFOAM (2020). The world of Organic Agriculture.Statistics and emerging trends. Disponible en: https://shop.fibl.org/CHen/mwdownloads/download/link/id/785/?ref=1 (Consulta realizada el 7 de diciembre de 2020).

Instituto Nacional de Estadística (INE) (2017). "Cifras oficiales de población de los municipios españoles: Revisión del Padrón Municipal". en: http://www.ine.es/dyngs/INEbase/es/operacion.htm?c=Estadistica_C\&cid=1254736177011\&menu=result ados\&idp=1254734710990 (Consulta realizada el 3 de diciembre de 2017).

SPSS, Inc. (2013). SPSS Categories. Version 22.0, Chicago. 